\title{
Deep convolutional neural network VGG-16 model for differential diagnosing of papillary thyroid carcinomas in cytological images: a pilot study
} \author{
Wan 2,4, , Jun Xiang 1,2, , \\ 1. Department of Head and Neck Surgery, Fudan University Shanghai Cancer Center, Shanghai, 200032, China \\ 2. Department of Oncology, Shanghai Medical Colloge, Fudan University, Shanghai, 200032, China \\ 3. Depertment of Computer Science and Engineering, Shanghai Jiaotong University, Shanghai, China \\ 4. Department of Pathology, Fudan University Shanghai Cancer Center, Shanghai, 200032, China \\ Qing Guan and Yunjun Wang contributed equally to the work and should be regarded as co-first authors.
}

Qing Guan1,2, Yunjun Wang1,2, Bo Ping2,4, Duanshu Li1,2, Jiajun Du³, Yu Qin³, Hongtao Lu³, Xiaochun

$\triangle$ Corresponding author: Jun Xiang, MD, phD, Department of Head and Neck Surgery, Fudan University Shanghai Carcinoma Center, Shanghai, 200032, China. Phone: +86 021-54175590 Ext. 65085; E-mail: junxiang82@163.com. Xiaochun Wan, MD, Department of Pathology, Fudan University Shanghai Carcinoma Center, Shanghai, 200032, China. E-mail: wach126@163.com.

(C) The author(s). This is an open access article distributed under the terms of the Creative Commons Attribution License (https://creativecommons.org/licenses/by/4.0/). See http://ivyspring.com/terms for full terms and conditions.

Received: 2018.07.25; Accepted: 2019.07.28; Published: 2019.08.27

\begin{abstract}
Objective: In this study, we exploited a VGG-16 deep convolutional neural network (DCNN) model to differentiate papillary thyroid carcinoma (PTC) from benign thyroid nodules using cytological images.

Methods: A pathology-proven dataset was built from 279 cytological images of thyroid nodules. The images were cropped into fragmented images and divided into a training dataset and a test dataset. VGG-16 and Inception-v3 DCNNs were trained and tested to make differential diagnoses. The characteristics of tumor cell nucleus were quantified as contours, perimeter, area and mean of pixel intensity and compared using independent Student's t-tests.

Results: In the test group, the accuracy rates of the VGG-16 model and Inception-v3 on fragmented images were $97.66 \%$ and $92.75 \%$, respectively, and the accuracy rates of VGG-16 and Inception-v3 in patients were $95 \%$ and $87.5 \%$, respectively. The contours, perimeter, area and mean of pixel intensity of PTC in fragmented images were more than the benign nodules, which were $61.01 \pm 17.10$ vs $47.00 \pm 24.08$, $p=0.000,134.99 \pm 21.42$ vs $62.40 \pm 29.15, p=0.000,1770.89 \pm 627.22$ vs $1157.27 \pm 722.23, p=0.013$, $165.84 \pm 26.33$ vs $132.94 \pm 28.73, p=0.000$ ), respectively.

Conclusion: In summary, after training with a large dataset, the DCNN VGG-16 model showed great potential in facilitating PTC diagnosis from cytological images. The contours, perimeter, area and mean of pixel intensity of PTC in fragmented images were more than the benign nodules.
\end{abstract}

Key words: Deep convolutional neural network, papillary thyroid carcinoma, cytological images, fine-needle aspiration, liquid-based cytology

\section{Introduction}

Thyroid nodules are a common medical condition, and most occurrences result in a benign outcome ${ }^{[1]}$. However, differential diagnosis of thyroid nodules is crucial because thyroid carcinoma requires surgery, but only follow-up care is required for benign nodules. An accurate evaluation of thyroid nodules is suggested by the American Thyroid
Association (ATA) guidelines along with recommendations about neck ultrasonography and fine-needle aspiration (FNA) cytology [2]. Cytological evaluation of an FNA biopsy specimen remains the most precise single test for evaluating thyroid nodules to detect potential carcinomas. Thyroid carcinoma has four major pathology types: papillary thyroid 
carcinoma, follicular thyroid carcinoma (FTC), medullary thyroid carcinoma (MTC) and anaplastic thyroid carcinoma (ATC). Among these, PTC is the most frequently diagnosed type ${ }^{[3]}$. The most common benign thyroid lesion is nodular goiter. Because FTC requires histology for definitive diagnosis and the incidences of MTC and ATC are relatively low, most studies concerning thyroid cytology choose to concentrate on differentiating PTC from benign nodules.

Applications that use machine learning (ML) have been increasing rapidly in the medical imaging field and have been applied to pathological diagnoses of various diseases [4-5]. The DCNN is a type of $\mathrm{ML}$ constructed using a special type of artificial neural network that resembles the multilayered human cognition system. Many research groups have investigated applications of DCNNs to pathological images [6-9]. Korbar et al. developed a system that used a DCNN to classify different types of colorectal polyps on whole-slide images [10]. Ertosun et al. proposed an automated system to grade gliomas using DCNN ${ }^{[11]}$. Most recently, an automated DCNN scheme was developed to classify lung carcinomas using cytological images. This system achieved excellent performance and ensured a promising role for DCNNs in cytological diagnosis [12].

Several studies have developed computer-assisted diagnostic systems for thyroid nodule cytology using models such as the Elman neural network (ENN) or the support vector machine (SVM) [13-15]. However, to our knowledge, a DCNN has not previously been applied to diagnose cytological images of thyroid nodules. In this study, we retrained two automated DCNN schemes to classify thyroid nodules with cytological images, which were VGG-16[16] and Inception-V3[17]. VGG-16 is a convolutional neural network model proposed by K. Simonyan and A. Zisserman from the University of Oxford, this model achieved $92.7 \%$ top-5 test accuracy in ImageNet, which is a dataset of over 14 million images belonging to 1000 classes $^{[16]}$. Inception-v3 is also a convolutional neural network that is trained on more than a million images from the ImageNet database. The training results of these two models were compared at the end of this study.

\section{Materials and Methods}

\section{Patients and cytological images}

This study was conducted with the approval of the ethics committee of Fudan University Shanghai Cancer Center (FUSCC). The methods were conducted in accordance with the approved guidelines. The cytological images required to develop and evaluate our method were collected from patients who underwent thyroid nodule FNA and thyroidectomy from January 1st, 2017 to July 31 st, 2017. Before the FNA procedure, written informed consent was obtained from all patients. An FNA biopsy was performed with 22-gauge needles by an experienced sonographer under ultrasonographic guidance. Thin layer liquid-based cytology (LBC) preparations are superior to conventional preparations with regard to background clarity, monolayer cell preparation and cell preservation. It is easier and less time consuming to screen and interpret LBC preparations because the cells are limited to smaller areas on clear backgrounds with excellent cellular preservation. Therefore, most of the FNA samples were transferred to a $10 \mathrm{ml}$ syringe and then prepared with a natural sedimentation-type thin layer LBC system using a BD SurePath liquid-based Pap Test (Beckton Dickinson, Durham, NC, USA). Moreover, because most cytological pathologists in China were more accustomed to hematoxylin-eosin (H\&E) staining, which can be easily compared with histological cell morphology, the LBC smears were usually processed with H\&E staining. A digital still camera (DP27, Olympus, Tokyo, Japan) with a $40 \times$ objective lens attached to a microscope (BX45, Olympus) was used to take the pictures for the LBC smears. All photos were collected by experienced cytopathologists and saved in JEPG format.

Our dataset contained 279 H\&E-stained images, each of which was associated with a different patient. The dataset included 159 cases of PTC and 120 cases of benign lesions. These slides were digitized at $400 \times$ magnification. Figure 1 shows some examples of the H\&E-stained PTC and benign thyroid nodule images used in this project. All the PTC images had classic features (including high cellularity, papillary fronds with anatomical edges, enlarged oval nuclei with longitudinal intranuclear grooves, nuclear crowding and overlapping, cellular swirls, and chewing gum colloid [18]) and were defined by our experienced cytopathologists as class V or VI according to the Bethesda system for reporting thyroid cytopathology [19]. All the selected patients underwent a thyroidectomy and were given a pathologic diagnosis of PTC. All the images of benign nodules fit the class II description in the Bethesda system and consisted of an adequately cellular specimen composed of varying proportions of colloid and benign follicular cells arranged as macrofollicles and macrofollicle fragments [19]. The patients with benign nodules did not receive surgery; thus, their histological data were not available. Consequently, the ground truth was derived from other clinical, laboratory, and imaging evaluations by an expert. 


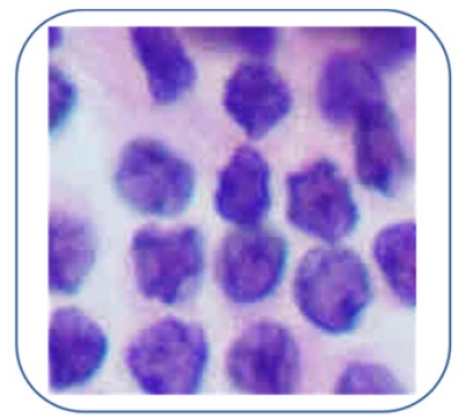

A

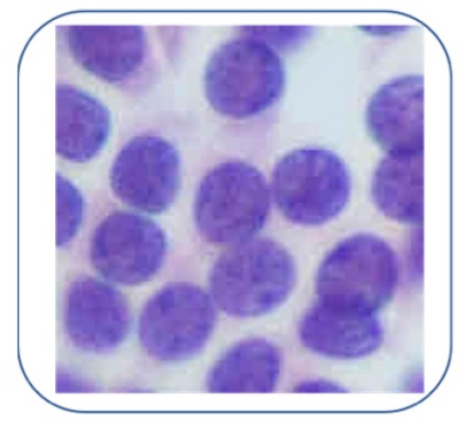

D

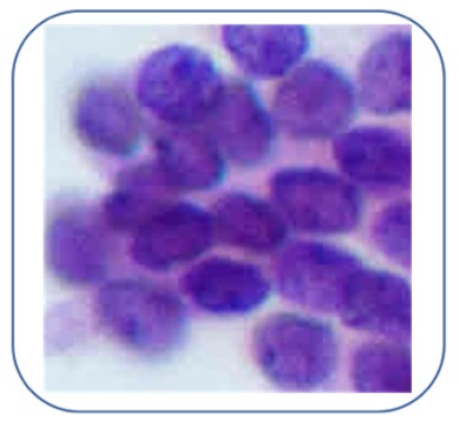

B

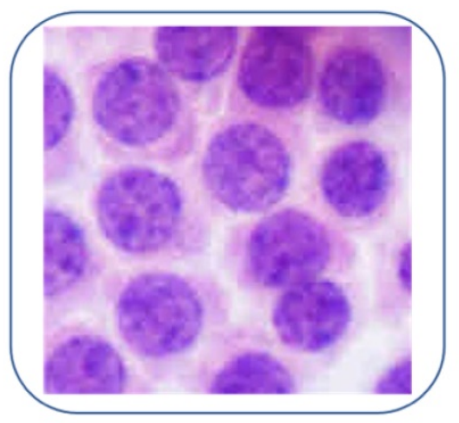

E

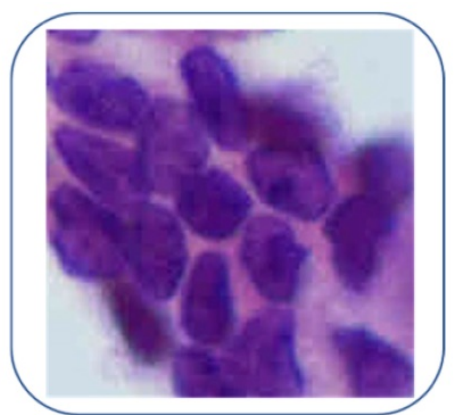

C

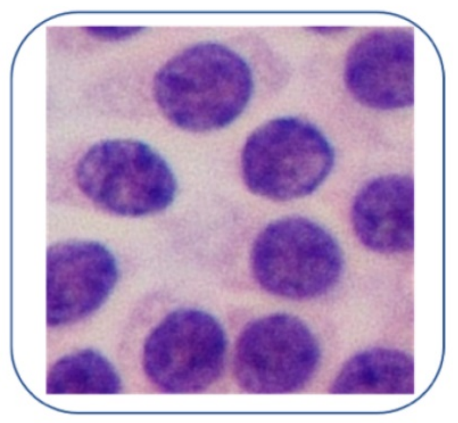

$\mathrm{F}$

Figure 1. Fragmented cytological images. (A, B, C): PTC; (D, E, F): benign nodules.

\section{Data augmentation}

In the dataset, each image is manually segmented into several $224 \times 224$ fragments that contain several cells. As shown in Table 1, this segmentation resulted in a total of $887224 \times 224$ fragmented images representing 476 PTC and 411 benign nodules. We split the dataset randomly into a training subset and a test subset for each cytology type at a ratio of approximately $6: 1$. We obtained 759 fragmented images for training and 128 fragmented images for testing with no overlap from the original images, resulting in 136 PTC and 103 benign nodule images in the training group and 23 PTC and 17 benign nodule whole-slide images in the test group.

Table 1. Number of fragmented images in the dataset.

\begin{tabular}{llll}
\hline Cytological type & Training data & Test data & Total \\
\hline PTC & 407 & 69 & 476 \\
Benign nodule & 352 & 59 & 411 \\
All type & 759 & 128 & 887 \\
\hline
\end{tabular}

We augmented the training data by flipping and rotating the images. Each image fragment was flipped horizontally and rotated by $0^{\circ}, 90^{\circ}, 180^{\circ}$ and $270^{\circ}$. Through this flipping and rotating process, we increased the size of the training data by 8 times. If we were instead to directly augment the training data, the required storage space would expand by 8 times.
Thus, to save storage space, we do not augment the training data in advance but only during the training process. In one iteration of the training process, we fetch a batch of images from the training data. We flip and rotate each image in the batch randomly. For each image, we randomly apply only one of the 8 transformation choices.

\section{Quantification of tumor cells}

In order to obtain the characteristics of the tumor cells, we decided to find the contour features in the image to quantify the characteristics of tumor cell nucleus. As shown in Figure 3, we first converted the tumor images to grayscale images (Figure 3B), then we used the Laplacian method to calculate the second partial derivative of the image pixels in order to obtain contours in each tumor image (Figure 3C). After we extracted all the cell contours in each tumor image, then we counted the number of contours in each image, and we calculated the perimeter and area of each contour, as well as the average value of pixel's intensity and standard deviation (SD) of each image. Independent Student's t-tests were used to compare those variables between PTC and benign nodules, a p-value less than 0.05 was considered significant.

\section{Network architecture}

We retrained two DCNN models in our experiment: VGG-16 and Inception-v3. The 
architecture of VGG-16 is shown in Table 2; it uses 13 convolutional layers and 3 fully connected layers. The convolutional layers in VGG-16 are all $3 \times 3$ convolutional layers with a stride size of 1 and the same padding, and the pooling layers are all $2 \times 2$ pooling layers with a stride size of 2 . The default input image size of VGG-16 is $224 \times 224$. After each pooling layer, the size of the feature map is reduced by half. The last feature map before the fully connected layers is $7 \times 7$ with 512 channels and it is expanded into a vector with $25,088 \quad(7 \times 7 \times 512)$ channels.

Table 2. Architecture of VGG-16 network

\begin{tabular}{lll}
\hline Layer & Patch size & Input size \\
\hline conv $\times 2$ & $3 \times 3 / 1$ & $3 \times 224 \times 224$ \\
pool & $2 \times 2$ & $64 \times 224 \times 224$ \\
conv $\times 2$ & $3 \times 3 / 1$ & $64 \times 112 \times 112$ \\
pool & $2 \times 2$ & $128 \times 112 \times 112$ \\
conv $\times 3$ & $3 \times 3 / 1$ & $128 \times 56 \times 56$ \\
pool & $2 \times 2$ & $256 \times 56 \times 56$ \\
conv $\times 3$ & $3 \times 3 / 1$ & $256 \times 28 \times 28$ \\
pool & $2 \times 2$ & $512 \times 28 \times 28$ \\
conv $\times 3$ & $3 \times 3 / 1$ & $512 \times 14 \times 14$ \\
pool & $2 \times 2$ & $512 \times 14 \times 14$ \\
$\mathrm{fc}$ & $25088 \times 4096$ & 25088 \\
$\mathrm{fc}$ & $4096 \times 4096$ & 4096 \\
\hline
\end{tabular}
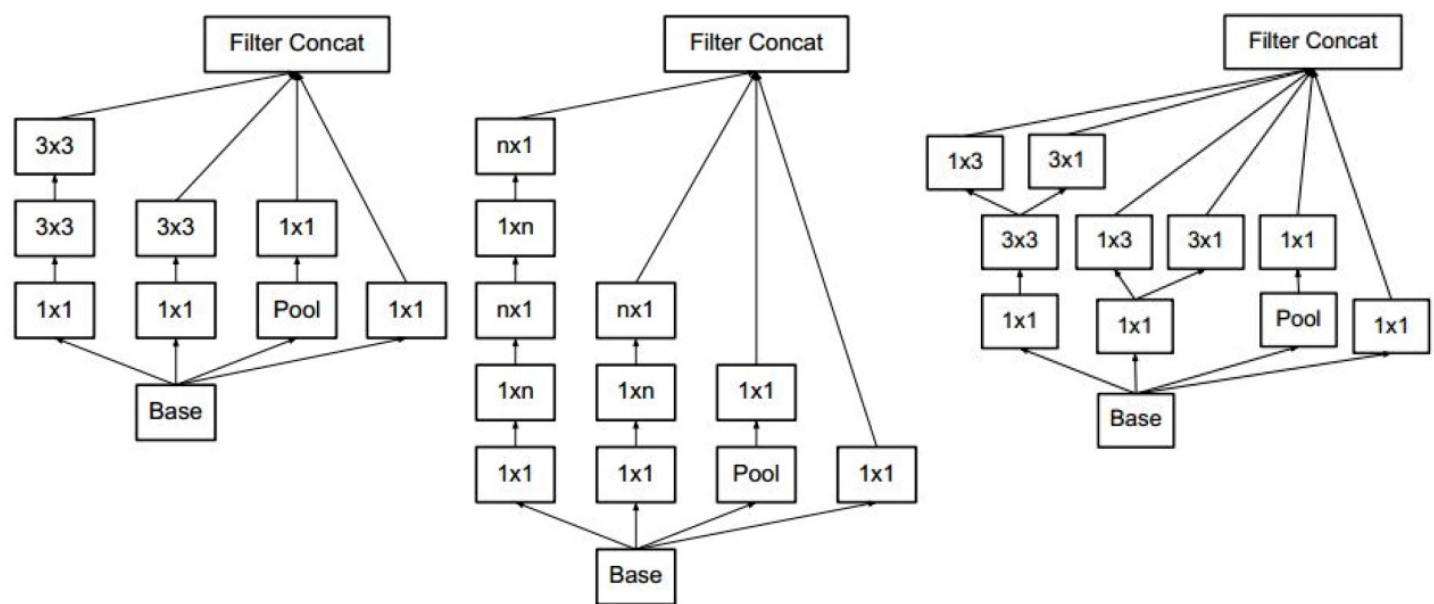

Conv stands for convolutional layer, pool stands for pooling layer and fc stands for fully connected layer. Patch size is the kernel size of convolutional layer, pooling layer or fully connected layer. Input size is feature map input size of the layer.

The layers of Inception-v3 are shown in Table 3. There were three types of Inception modules in the Inception-v3 model, as shown in Figure 2 (from left to right, Inception modules A, B and C). The Inception modules are well-designed convolution modules that both generate discriminatory features and reduce parameters. Each Inception module is composed of several convolutional and pooling layers in parallel. Small convolutional layers (e.g., $3 \times 3,1 \times 3,3 \times 1$, and $1 \times 1)$ are used in the Inception modules to reduce the number of parameters. Inception-v3 stacks 3 Inception A, 5 Inception B and 2 Inception C modules in series. The default input image size for the VGG-16 model is $229 \times 229$. After the convolutional and Inception modules, the resulting feature map is $8 \times 8$ with 2,048 channels. We used a global pooling layer before the fully connected layer.

Figure 2. The Inception modules of Inception-v3. The Inception modules of Inception-v3 including Inception module A, B and C from left to right. Each Inception module is composed of several convolutional layers and pooling layers. Pool stands for pooling layer and $\mathrm{n} \times \mathrm{m}$ stands for $\mathrm{n} \times \mathrm{m}$ convolutional layer.

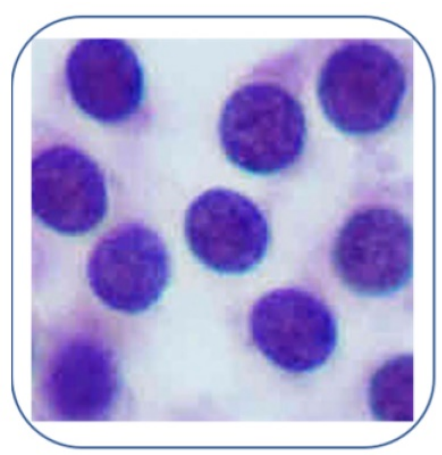

A

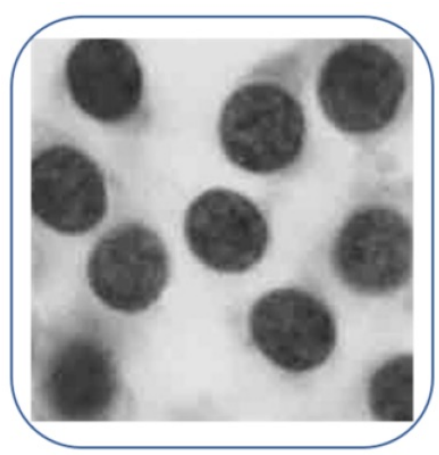

B

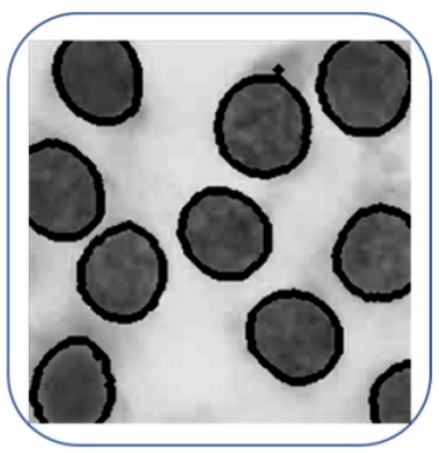

C

Figure 3. Quantification of tumor cells. (A) Original images, (B) Grayscale images, (C) contours of tumor cells. 
Table 3. Architecture of Inception-v3 network

\begin{tabular}{lll}
\hline Layer & Patch size & Input size \\
\hline conv & $3 \times 3 / 2$ & $229 \times 229 \times 3$ \\
conv & $3 \times 3 / 1$ & $149 \times 149 \times 32$ \\
conv padded & $3 \times 3 / 1$ & $147 \times 147 \times 32$ \\
pool & $3 \times 3 / 2$ & $147 \times 147 \times 64$ \\
conv & $3 \times 3 / 1$ & $73 \times 73 \times 64$ \\
conv & $3 \times 3 / 2$ & $71 \times 71 \times 80$ \\
conv & $3 \times 3 / 1$ & $35 \times 35 \times 192$ \\
Inception A×3 & ----- & $35 \times 35 \times 288$ \\
Inception B $\times 5$ & ----- & $17 \times 17 \times 768$ \\
Inception C×2 & ----- & $8 \times 8 \times 1280$ \\
pool & $8 \times 8$ & $8 \times 8 \times 2048$ \\
linear & logits & 2048 \\
softmax & classifier & 2 \\
\hline
\end{tabular}

Conv stands for convolutional layer, pool stands for pooling layer and fc stands for fully connected layer. Patch size is the kernel size of convolutional layer, pooling

layer or fully connected layer. Input size is feature map input size of the layer.

Table 4. Diagnostic efficiency of VGG-16 and Inception-v3 on test data (fragmented images)

\begin{tabular}{lll}
\hline Model & VGG-16 & Inception-v3 \\
\hline Accuracy & $97.66 \%$ & $92.75 \%$ \\
Sensitivity & $100 \%$ & $98.55 \%$ \\
Specificity & $94.91 \%$ & $86.44 \%$ \\
Positive predictive value & $95.83 \%$ & $89.47 \%$ \\
Negative predictive value & $100 \%$ & $98.08 \%$ \\
\hline
\end{tabular}

The output of the original networks of Inception-v3 and VGG-16 includes 1,000 classes, but our case required only 2 classes, PTC and benign nodules. Therefore, we changed the output channel number of the last layer from 1,000 to 2 . We also used a dropout rate of $50 \%$ during the training process. The dropout process randomly discards some layer inputs and is used to avoid overfitting.

We used the pretrained models offered by TensorFlow and finetuned them using cytological images. The models were pretrained on the ImageNet dataset and can be found in the TensorFlow-Slim image classification library. We initialized the parameters from the pretrained model because ImageNet had approximately 14,000,000 images, but we had only 279 images. It was difficult to train the models with such a small number of images because deep networks have a large number of parameters. Pretraining can speed up network convergence.

\section{Results}

We used both VGG-16 and Inception-v3 in the experiments. The default image size of VGG-16 was $224 \times 224$, which was the same as the sizes of the images in the dataset; however, the default image size of Inception-v3 was $299 \times 299$. Consequently, we resized the images to $299 \times 299$ when using Inception-v3 to train and test. We trained the two models on the training data and tested them on the test data. Table 4 shows the models' diagnostic efficiency of the test data. Using the VGG-16 model, the DCNN achieved an accuracy of $97.66 \%$ on the fragmented images; however, among the three misdiagnosed images, two belonged to one patient; therefore, two patients in the test group received false positively diagnoses by the DCNN. From a patient-wise viewpoint, the accuracy rate was $95 \%$ (38/40). Using the Inception-v3 model, the results included nine misdiagnosed fragmented images, among which 5 patients were given a false positively diagnosis. The one false negative diagnosis would not affect the patient's final diagnosis; thus, the total accuracy rate for Inception-v3 was $87.5 \%$ (35/40). In these experiments, the VGG-16 results were much better than those of the Inception-v3 model.

The data of quantification were shown in Table 5, firstly, the contours of malignant tumors in fragmented images were more than the benign tumors $(61.01 \pm 17.10$ vs $47.00 \pm 24.08, \mathrm{p}<0.001)$, which reflected the fact that PTC tumor cells were more crowded on the cytological images. Secondly, the perimeter $(134.99 \pm 21.42$ vs $62.40 \pm 29.15, \mathrm{p}<0.001)$ and area $(1770.89 \pm 627.22$ vs $1157.27 \pm 722.23, \mathrm{p}=0.013)$ of each PTC cell nucleus were also bigger in the PTC indicating that nuceus of PTC were larger than the benign ones. Last but not least, the means of pixel intensity $(165.84 \pm 26.33$ vs $132.94 \pm 28.73, \mathrm{p}<0.001)$ were higher in PTC, which suggested that PTC nuceus have stronger staining.

Table 5. Quantification of tumor cells in fragmented images of malignant and benign thyroid tumors.

\begin{tabular}{llll}
\hline & Malignant & Benign & p value \\
\hline Contour & $61.01 \pm 17.10$ & $47.00 \pm 24.08$ & $<0.001$ \\
Perimeter & $134.99 \pm 21.42$ & $62.40 \pm 29.15$ & $<0.001$ \\
Area & $1770.89 \pm 627.22$ & $1157.27 \pm 722.23$ & 0.013 \\
Mean of pixel intensity & $165.84 \pm 26.33$ & $132.94 \pm 28.73$ & $<0.001$ \\
\hline
\end{tabular}

After the comparison, we chose VGG-16 over Inception-v3 for further investigation. We further investigated the misdiagnosed images to analyze the reasons for failure. Figure 4 shows the three misdiagnosed fragmented images: note that Figure 4B and Figure $4 \mathrm{C}$ were cropped from the same image. The cytopathologists who reviewed these three images considered them to be typical benign nodules, however, the quantification data indicated that those images were more like PTC except the contours (Figure 4A: contour 17, perimeter 149.67, area 1685.91, mean pixel intensity 163.47; Figure 4B, Contour 19, perimeter 107.17 , area 1469.83 , mean pixel intensity 165.35; Figure 4C, Contour 21, perimeter 127.00, area 1839.65 , mean pixel intensity 182.79 ).

\section{Discussion}

Deep learning has a good performance in image classification, in recent years, a series of deep learning models have been applied to image classification, 


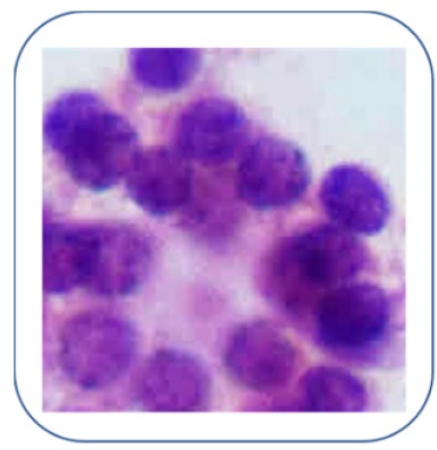

A

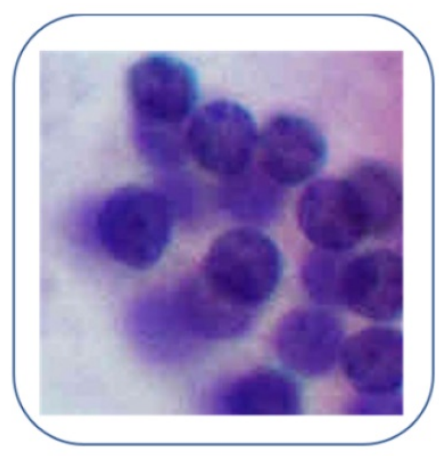

B

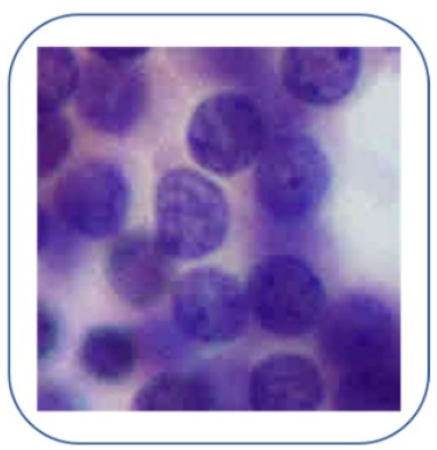

C

Figure 4. Misdiagnosed fragmented images. (B) and (C) were cropped from same image. A: contour 17, perimeter 149.67, area 1685.91, mean pixel intensity 163.47; B: Contour 19, perimeter 107.17, area 1469.83, mean pixel intensity 165.35; C: Contour 21, perimeter 127.00, area 1839.65, mean pixel intensity 182.79.

such as AlexNet, VGGNet and InceptionNet. VGG-16 is a deep convolutional neural network consist of 16 layers that is combined by many $3 \times 3$ convolutional layers and $2 \times 2$ pooling layers repeatedly, and VGG-16 has a remarkable feature extraction's capability so that it can obtain a good effect in image classification. VGG-16 has a better feature learning ability than AlexNet because it's deeper than AlexNet and it can get more sparse features than AlexNet. Because VGG-16 just uses $3 \times 3$ convolution layer and $2 \times 2$ pooling layer repeatedly, so that VGG-16 is relatively simpler than InceptionNet, so it has a better generalization's ability, and it can adapt to a variety of data sets including tumor images. As for InceptionNet, there are different sizes of convolution kernels in InceptionNet, so IncetionNet is more suitable for multi-size target's classification, but our tumor images are collected at the same resolution, so InceptionNet is not suitable. In our experiment, VGG-16 achieved an accuracy rate was 95\% (38/40) patient wisely, while the accuracy rate was $95 \%$ $(38 / 40)$ for Inception-v3, which confirmed the advantage of VGG-16 in tumor image classification.

FNA cytology is a well-accepted method for diagnosing PTC, and it has an estimated accuracy of approximately $89-95 \%$ [20]. With the VGG-16 model and selected patients, DCNN achieved 97.66\% accuracy in fragmented images and a patient-wise accuracy rate of $95 \%$. Previously, Teramoto A et al developed an automated scheme of DCNN to classify adenocarcinoma, squamous cell carcinoma (SCC), and small cell carcinoma in lung cancer using cytological images, the accuracy rate of classification was 70\% [12]. Momeni-Boroujeni et al reported a study using multilayer neural network (MNN) to distinguish benign from malignant pancreatic nodules using cytological images, which achieved 100\% accuracy, and it can categorize atypical cases into benign or malignant with $77 \%$ accuracy ${ }^{[21]}$. Compares to these studies, our results were quite satisfactory, however, there is still room for improvement. It is also worth mentioning that the VGG-16 model did not result in any false negative diagnoses, which indicates that it may be a candidate for use as a screening tool to reduce cytopathologists' workloads. Because computer screening systems have already been introduced to the practice of cervical cytology [22], further study is warranted to validate their application in thyroid or other types of disease.

In this study, we also analyzed misdiagnosed fragmented images, all three images were from benign nodules and were misdiagnosed as PTC. Although the quantification data showed that the perimeter, area and means of pixel intensity of those images were close to PTC, our cytopathologists considered those images to be typical benign nodules. In that case we assumed that the DCNN was making the diagnosis based on the size and staining of the nucleus but not the shape of it, future study should work a way for training the network to differentiate the cellular and nucleus morphology.

The cytological images used in this study all matched the Bethesda classes II, V and VI and included only the PTC pathology subtype. Because it has now been demonstrated that a DCNN can be used to differentiate PTC from benign nodules, future studies should investigate its use in Bethesda class III and IV images, which show atypia of undetermined significance or follicular lesions of undetermined significance, follicular neoplasm or are suspicious for a follicular neoplasm [19]. Since this is a pilot study, we have only included a few typical cytological images, in the future study, we could expand the sample size from our hospital or search cooperation with another institution, and there is a chance that we may post our system online so that users can conduct their own assessment. 


\section{Conclusions}

In summary, after training with a large dataset, the VGG-16 model of DCNN showed great potential for facilitating the PTC diagnoses using cytological images. The contours, perimeter, area and mean of pixel intensity of PTC in fragmented images were more than the benign nodules.

\section{Abbreviations}

DCNN: deep convolutional neural network; PTC: papillary thyroid carcinoma; ATA: American Thyroid Association; FNA: fine-needle aspiration; FTC: follicular thyroid carcinoma; MTC: medullary thyroid carcinoma; ATC: anaplastic thyroid carcinoma; ML: machine learning; SVM: support vector machine; FUSCC: Fudan University Shanghai Cancer Center; LBC: liquid-based cytology; H\&E: hematoxylin-eosin; SCC: squamous cell carcinoma; MNN: multilayer neural network.

\section{Acknowledgements}

This study was funded by the Shanghai Municipal Planning Commission of Science and Research Funds for Young Scholars (award number 20154Y0050) and National Nature Science Foundation of China (No.61772330, 61533012, 61876109).

\section{Author Contributions}

Qing Guan and Yunjun Wang analyzed the data and wrote and edited the original draft; Jun Xiang designed the study, directed and coordinated the project, curated the data, and edited the draft; Xiaochun Wan and Bo Ping performed cytopathology diagnoses, collected pictures, and edited the draft; Duanshu Li analyzed the data helped write the original draft and directed and coordinated the project; Hongtao Lu, Jiajun $\mathrm{Du}$ and Yu Qin were responsible for software validation and data curation.

\section{Competing Interests}

The authors have declared that no competing interest exists.

\section{References}

[1] Siegel R, Naishadham D, Jemal A. Cancer statistics, 2012. Ca Cancer J Clin.2012;62:10-29.

[2] Haugen BR, Alexander EK, Bible KC, et al. 2015 American Thyroid Association Management Guidelines for Adult Patients with Thyroid Nodules and Differentiated Thyroid Cancer: The American Thyroid Association Guidelines Task Force on Thyroid Nodules and Differentiated Thyroid Cancer. Thyroid.2016;26:1-133.

[3] La Vecchia C, Malvezzi M, Bosetti C, et al. Thyroid cancer mortality and incidence: a global overview. Int J Cancer.2015;136:2187-95. [4] He L, Long LR, Antani S. Histology image analysis for carcinoma detection and grading. Comput Methods and Programs Biomed. 2012 2012;107:538- 56.

[5] Barker J, Hoogi A, Depeursinge A, et al. Automated classification of brain tumor type in whole-slide digital pathology images using local representative tiles. Med Image Anal.2016;30:60-71.
[6] Miki Y, Muramatsu C, Hayashi T, et al. Classifcation of teeth in cone-beam CT using deep convolutional neural network. Comput Biol Med.2017;80:24-9.

[7] Cha KH, Hadjiiski L, Samala RK, et al. Urinary bladder segmentation in CT urography using deep-learning convolutional neural network and level sets. Med Phys.2016;43:1882-6.

[8] Anthimopoulos M, Christodoulidis S, Ebner L, et al. Lung Pattern Classification for Interstitial Lung Diseases Using a Deep Convolutional Neural Network. IEEE Trans Med Imaging.2016;35:1207-16.

[9] Teramoto A, Fujita H, Yamamuro O, et al. Automated detection of pulmonary nodules in PET/CT images: Ensemble false-positive reduction using a convolutional neural network technique. Med Phys.2016;43:2821-7.

[10] Korbar B, Olofson AM, Miraflor AP, et al. Deep learning for classification of colorectal polyps on whole -slide images. J Pathol Inform.2017;25:30.

[11] Ertosun MG, Rubin DL. Automated grading of gliomas using deep learning in digital pathology images: A modular approach with ensemble of convolutional neural networks. AMIA Annu Symp Proc.2015. 2015: 1899-1908.

[12] Teramoto A, Tsukamoto T, Kiriyama Y, et al. Automated Classification of Lung Carcinoma Types from Cytological Images Using Deep Convolutional Neural Networks. Biomed Res Int.2017;2017:4067832.

[13] Gopinath B, Shanthi N. Support Vector Machine based diagnostic system for thyroid carcinoma using statistical texture features. Asian Pac J Carcinoma Prev.2013;14:97-102.

[14] Gopinath B, Shanthi N. Computer-aided diagnosis system for classifying benign and malignant thyroid nodules in multi-stained FNAB cytological images. Australas Phys Eng Sci Med.2013;36:219-30.

[15] Gopinath B, Shanthi N. Development of an automated medical diagnosis system for classifying thyroid tumor cells using multiple classifier fusion. Technol Carcinoma Res Treat.2015;14:653-62.

[16] Simonyan K, Zisserman A. Very deep convolutional networks for large-scale image recognition. arXiv. 2014;arXiv.1409.1556.

[17] Szegedy C, Vanhoucke V, Ioffe S, et al. Rethinking the Inception architecture for computer vision[C]//Proceedings of the IEEE Conference on Computer Vision and Pattern Recognition. 2016; 2818-26.

[18] Akhtar M, Ali MA, Huq M, et al. Fine needle aspiration biopsy of papillary thyroid carcinoma: Cytologic, histologic and ultrastructural correlations. Diagn Cytopathol.2010;7:373-9.

[19] Cibas ES, Ali SZ. The Bethesda system for reporting thyroid cytopathology. Thyroid.2009;19:1159-65.

[20] Bongiovanni M, Spitale A, Faquin WC, et al. The Bethesda System for Reporting Thyroid Cytopathology: a meta-analysis. Acta Cytol 2012;56:333-9.

[21] Momeni-Boroujeni A, Yousefi E, Somma J. Computer-Assisted Cytologic Diagnosis in Pancreatic FNA: An Application of Neural Networks to Image Analysis. Cancer Cytopathol.2017;125:926-33.

[22] Pantanowitz L, Hornish M, Goulart RA. The impact of digital imaging in the field of cytopathology. Cytojournal.2009;6:59-70. 